\title{
Periodic boundary condition induced breakdown of the equipartition principle and other kinetic effects of finite sample size in classical hard-sphere molecular dynamics simulation
}

\author{
Randall B. Shirts \\ randy_shirts@byu.edu \\ Scott R. Burt \\ scott_burt@byu.edu \\ Aaron M. Johnson
}

Follow this and additional works at: https://scholarsarchive.byu.edu/facpub

Part of the Biochemistry Commons, and the Chemistry Commons

\section{Original Publication Citation}

Shirts, Randall B., Scott R. Burt, and Aaron M. Johnson."Periodic boundary condition induced breakdown of the equipartition principle and other kinetic effects of finite sample size in classical hard-sphere molecular dynamics simulation." The Journal of

\section{BYU ScholarsArchive Citation}

Shirts, Randall B.; Burt, Scott R.; and Johnson, Aaron M., "Periodic boundary condition induced breakdown of the equipartition principle and other kinetic effects of finite sample size in classical hard-sphere molecular dynamics simulation" (2006). Faculty Publications. 286.

https://scholarsarchive.byu.edu/facpub/286

This Peer-Reviewed Article is brought to you for free and open access by BYU ScholarsArchive. It has been accepted for inclusion in Faculty Publications by an authorized administrator of BYU ScholarsArchive. For more information, please contact ellen_amatangelo@byu.edu. 


\title{
Periodic boundary condition induced breakdown of the equipartition principle and other kinetic effects of finite sample size in classical hard-sphere molecular dynamics simulation
}

\author{
Randall B. Shirts, ${ }^{\text {a) }}$ Scott R. Burt, ${ }^{\text {b) }}$ and Aaron M. Johnson ${ }^{\text {b) }}$ \\ Department of Chemistry and Biochemistry, Brigham Young University, Provo, Utah 84602-5700
}

(Received 23 May 2006; accepted 11 September 2006; published online 24 October 2006)

\begin{abstract}
We examine consequences of the non-Boltzmann nature of probability distributions for one-particle kinetic energy, momentum, and velocity for finite systems of classical hard spheres with constant total energy and nonidentical masses. By comparing two cases, reflecting walls ( $N V E$ or microcanonical ensemble) and periodic boundaries (NVEPG or molecular dynamics ensemble), we describe three consequences of the center-of-mass constraint in periodic boundary conditions: the equipartition theorem no longer holds for unequal masses, the ratio of the average relative velocity to the average velocity is increased by a factor of $[N /(N-1)]^{1 / 2}$, and the ratio of average collision energy to average kinetic energy is increased by a factor of $N /(N-1)$. Simulations in one, two, and three dimensions confirm the analytic results for arbitrary dimension. (C) 2006 American Institute of Physics. [DOI: 10.1063/1.2359432]
\end{abstract}

\section{INTRODUCTION}

The equipartition theorem is a fundamental result of classical statistical mechanics and states, loosely, that each classical degree of freedom equally shares the system energy. In other words, each degree of freedom is at the same temperature. For a molecular dynamics simulation with periodic boundaries, this paper shows that, as a result of the conservation of total linear momentum, the equipartition theorem breaks down for a finite, hard-sphere system when particles have different masses. Light particles have a higher equilibrium average energy than heavy particles, and, in fact, the entire equilibrium one-particle energy distribution depends on particle mass. These differences are inversely proportional to the total mass of the system. We also show that, for a similar reason, even for equal masses, the ratio of average relative velocity to average velocity is increased by a factor of $[N /(N-1)]^{1 / 2}$, and the ratio of average collision energy to average kinetic energy is increased by a factor of $N /(N-1)$.

Hard spheres have served for a century as a powerful model system to elucidate general statistical principles because analytic results are often possible. As a pedagogical tool, we previously developed a graphical two-dimensional hard-sphere simulation code for display by wINDOws computers. This software has been available since 1995 as the software package BOLTZMANN (Ref. 1) and has proven very useful in helping students visualize the meaning of the Maxwell-Boltzmann velocity distribution, the Boltzmann energy distribution, and free path and collision time distributions, among others. An improved, more recent downloadable JAVA application, BOLTZMANN 3D, ${ }^{2}$ performs hard-sphere simulations in one, two, or three dimensions and graphically

\footnotetext{
a) Author to whom correspondence should be addressed. Electronic mail: randy_shirts@byu.edu

${ }^{b}$ Present address; Department of Chemistry, University of California, Berkeley, CA 94720-1460.
}

displays the motion on the computer screen in real time. If one compares the averages predicted by the Boltzmann distribution, the Maxwell-Boltzmann velocity distribution, and other simple predictions of kinetic theory with the results of hard-sphere simulations in the BOLTZMANN, BOLTZMANN 3D, or similar simulations, consistent differences are noted for small numbers of particles. The results derived here explain these discrepancies and identify several complications not previously documented. These complications will occur also, although probably less obviously, in molecular dynamics simulations with more realistic attractive potentials.

The results presented here should be of interest to researchers doing either statistical mechanics or molecular dynamics simulation. It is often assumed that the velocity distribution in a simulation should be a Maxwell-Boltzmann distribution with a well-defined temperature and that the use of periodic boundary conditions is an easy method to mimic the behavior of a bulk system. Our results for hard spheres demonstrate how surprising even simple systems can be. The implicit conservation of linear momentum in a system with periodic boundary conditions actually makes the system behave like a smaller system ( $N-1$ vs $N$ particles) and disrupts even cherished principles like equipartition. The fact that finite hard-sphere systems exhibit a distinctly non-MaxwellBoltzmann velocity distribution may not be new to some, but the fact that particles with different masses have differing equilibrium distributions (with different average energies and, thus, different temperatures) in the same equilibrium simulation will probably be surprising to most. These finite size effects give rise to corrections of the order of $N^{-1}$ in temperature, collision rates, mean free paths, equation of state, and reaction probability. These corrections should be accounted for in extrapolating to bulk properties; however, the nature of these corrections for systems with attractive potentials is still under investigation. 
In a previous publication, ${ }^{3}$ Shirts demonstrated using several simple quantum systems as examples that the probability distribution of one-particle energies in a small microcanonical quantum system ( $N V E$ ensemble) falls to zero considerably faster than the Boltzmann distribution for sufficiently large energy. This result is primarily a consequence of the fixed total energy of the $N V E$ ensemble; it is extremely unlikely for any one particle to accumulate a large portion of the total energy. Deviations from the Boltzmann distribution are inversely proportional to the size of the system, so the Boltzmann distribution is regained in the infinite system limit. However, these unusual properties would be measurable in atomic scale physical phenomena such as cluster dynamics, nuclear decay, and unimolecular reactions.

Probability distributions in classical microcanonical systems such as molecular dynamics simulations exhibit properties similar to those of quantum systems. The purpose of the present article is to explore some consequences of finite particle number and the associated energy and momentum constraints. It is well known that different statistical mechanical ensembles differ by corrections of the order of $N^{-1}$, where $N$ is the number of particles. ${ }^{4}$ We demonstrate several new order $N^{-1}$ corrections and discuss when they apply so that molecular dynamics simulations and experiments on isolated nanoscale systems can be accurately interpreted.

In the following sections, we first present results for the $N V E$, or microcanonical, ensemble appropriate for a container with reflecting walls. Then, we present results for the NVEPG, or microcanonical molecular dynamics, ensemble that is appropriate for periodic boundary conditions, where the total linear momentum $\boldsymbol{P}$ and the center-of-mass position $\boldsymbol{G}$ are also conserved. Breakdown of the equipartition principle as well as $N^{-1}$ corrections in average relative velocity and average collision energy are understood only by comparing results for the two ensembles. We use the term hard sphere to generically refer to hard spheres (three dimensions), hard disks (two dimensions), and hard rods (one dimension), but the results are generalizable to higher dimensions as well.

The analytic distributions displayed in subsequent sections are compared to results from computer simulations of $N$ hard-sphere particles in a $d$-dimensional volume $V_{d}$ and constant total energy $E$ with either reflecting walls or periodic boundary conditions. We have performed simulations on hard spheres for $d=1-3$ with both types of boundary conditions using $N$ from 3 to as many as 1000 particles. Our procedure for time propagation is similar to that described by Erpenbeck and Wood ${ }^{5}$ or Allen and Tildesley. ${ }^{6}$ We store time-weighted averages of moments $(k=1,2,4,-1 / 2)$ of one-particle energy $E_{i}$, velocity $v_{i}$, and other quantities. Also, for each set of particles, a set of equal-width intervals (bins) is established to accumulate statistics of the time-weighted distribution of $E_{i}, E_{i}^{2}, \nu_{i}, \nu_{i}^{2}$, etc. Some of these simulation statistics will be presented below in confirmation of theoretical predictions as they are developed. Additional examples are accessible online. ${ }^{7}$

\section{THE MICROCANONICAL ENERGY DISTRIBUTION}

The one-particle energy distribution function for a finite number of classical particles ( $N V E$ ensemble) can be derived, following Schlüter, ${ }^{8}$ in terms of $\Omega_{d}\left(N, V_{d}, E\right)$, the number of classical states below an energy $E$ for a $d$-dimensional system of $N$ particles of masses $m_{i}, i=1,2, \ldots, N$, in a $d$-dimensional volume $V_{d}$. In this case, $\Omega_{d}\left(N, V_{d}, E\right)$ is the volume contained by the energy shell $\boldsymbol{H}=E$ in $2 d N$-dimensional phase space,

$$
\begin{aligned}
\Omega_{d}\left(N, V_{d}, E\right) & =h^{-d N} \int_{H \leqslant E} d q^{d N} d p^{d N} \\
& =h^{-d N} \int \Theta(E-\boldsymbol{H}) d q^{d N} d p^{d N},
\end{aligned}
$$

where $\Theta(x)$ is the Heaviside step function, and we have chosen Planck's constant $h$ as the scale factor for classical states for proper dimensions and to maintain approximate correspondence with quantum state counting. We assume distinguishable particles, but products of factorials could be added to the denominator of Eq. (1) for sets of indistinguishable particles.

The density of states is given by

$$
\begin{aligned}
G_{d}\left(N, V_{d}, E\right) & =\left(\frac{\partial \Omega_{d}\left(N, V_{d}, E\right)}{\partial E}\right)_{N, V_{d}} \\
& =h^{-d N} \int \delta(E-\boldsymbol{H}) d q^{d N} d p^{d N},
\end{aligned}
$$

where $\delta(x)$ is the Dirac delta function.

We follow Schlüter ${ }^{8}$ and Çagin and Ray ${ }^{9}$ in defining the entropy of the $N V E$ ensemble as $S=k_{B} \ln \Omega_{d}$, where $k_{B}$ is Boltzmann's constant. They justify using $\Omega_{d}$ as the argument of the logarithm, rather than $G_{d}$ as is often done, ${ }^{10-12}$ using the principle of invariance of phase volumes to changes in external parameters. ${ }^{13}$ Thermodynamic functions for the $N V E$ ensemble, as well as for the NVEPG ensemble discussed below, are described in terms of the derivatives of $\Omega_{d \cdot}{ }^{14}$ In particular, the temperature is defined by

$$
T^{-1}=\left(\frac{\partial S}{\partial E}\right)_{V}=\frac{k_{B}}{\Omega_{d}}\left(\frac{\partial \Omega_{d}}{\partial E}\right)_{V}=\frac{k_{B} G_{d}}{\Omega_{d}} .
$$

The integral over momenta in Eq. (1) can be performed as the volume of a $d N$-dimensional hyperellipsoid ${ }^{15}$ with semiaxes $\left[2(E-U) m_{i}\right]^{1 / 2}$,

$$
\begin{aligned}
\Omega_{d}\left(N, V_{d}, E\right)= & \frac{2}{h^{d N} d N \Gamma(d N / 2)}\left(\prod_{i=1}^{N} m_{i}^{d / 2}\right) \\
& \times \int[2 \pi(E-U)]^{d N / 2} \Theta(E-U) d q^{d N}, \\
= & \frac{Z\left(N, V_{d}\right)}{h^{d N}}\left(\prod_{i=1}^{N} m_{i}^{d / 2}\right) \frac{2(2 \pi E)^{d N / 2}}{d N \Gamma(d N / 2)} \quad(\mathrm{hs}),
\end{aligned}
$$

where $\Gamma(x)$ is the gamma function, $Z\left(N, V_{d}\right)$ is the hardsphere configurational integral, and where we have used $U=0$ wherever the integrand of Eq. (4) does not vanish (im- 
penetrable bodies) in arriving at the last equality. The prefix hs in front of the equation number hereafter indicates that the equation applies only to hard spheres.

The kinetic definition of temperature for the $N V E$ ensemble is obtained using the same method as Çagin and Ray ${ }^{9}$ and Pearson et al., ${ }^{13}$

$$
\begin{aligned}
\langle K\rangle_{N V E} & =\frac{d}{2} N k_{B} T, \\
& =E \quad(\mathrm{hs}) .
\end{aligned}
$$

Note that Eq. (6) is an exact definition for any number of particles with any potential energy function, not just in the thermodynamic limit. Equation (7) uses $U=0$ in accessible configurations for hard spheres but is still an exact definition for any number of hard spheres.

The normalized one-particle momentum distribution can be obtained by integrating the phase space density $\delta(E$ $-\boldsymbol{H}) h^{-d N} / G_{d}\left(N, V_{d}, E\right)$ over all coordinates and all momenta except those of particle 1 . This integration is facilitated by expressing the $\delta$ function as a Fourier or Laplace transform. ${ }^{13,16}$ The result is ${ }^{17}$

$$
\begin{aligned}
P_{d}\left(N, V_{d}, E, \boldsymbol{p}_{1}\right) d \boldsymbol{p}_{1}^{d}= & \frac{\Gamma(d N / 2)}{\Gamma(d(N-1) / 2)\left(2 \pi m_{1} E\right)^{d / 2}} \\
& \times\left(1-\frac{\boldsymbol{p}_{1}^{2}}{2 m_{1} E}\right)^{[d(N-1) / 2]-1} \\
& \times \Theta\left(E-\frac{\boldsymbol{p}_{1}^{2}}{2 m_{1}}\right) d \boldsymbol{p}_{1}^{d} \quad \text { (hs). }
\end{aligned}
$$

The step function enters this expression because the integration contour is closed in the left-hand plane for $E_{1}<E$, enclosing the pole at $z=0,{ }^{18}$ while the contour must be closed in the right half plane for $E_{1}>E$. Converting to kinetic energy, $E_{1}=p_{1}^{2} /\left(2 m_{1}\right)$, the probability of a particle arbitrarily labeled 1 having a kinetic energy between $E_{1}$ and $E_{1}+d E_{1}$ in a $d$-dimensional $N V E$ system of $N$ hard-sphere particles is given by ${ }^{19,20}$

$$
\begin{aligned}
P_{d}\left(N, V_{d}, E, E_{1}\right) d E_{1}= & \frac{\Gamma(d N / 2)}{\Gamma(d / 2) \Gamma(d(N-1) / 2)}\left(\frac{E_{1}}{E}\right)^{d / 2} \\
& \times\left(1-\frac{E_{1}}{E}\right)^{[d(N-1) / 2]-1} \\
& \times \Theta\left(E-E_{1}\right) \frac{d E_{1}}{E_{1}} \quad \text { (hs). }
\end{aligned}
$$

For low energy, the function $P_{d}\left(N, V_{d}, E, E_{1}\right)$ initially rises as $E_{1}^{d / 2-1}$ according to the one-particle density of states, but as $E_{1}$ approaches the total energy $E, P_{d}\left(N, V_{d}, E, E_{1}\right)$ vanishes algebraically as a power of $E-E_{1}$. The corresponding Boltzmann distribution is obtained by taking the limit $N \rightarrow \infty$ and $E \rightarrow \infty$ with $E / N=d k_{B} T / 2$ according to Eq. (7),

$$
P_{B}\left(E_{1}\right)=\frac{(k T)^{-d / 2}}{\Gamma(d / 2)} E_{1}^{d / 2-1} e^{-E_{1} / k T} .
$$

The Boltzmann distribution decays to zero exponentially but remains nonzero as energy increases without bound. The

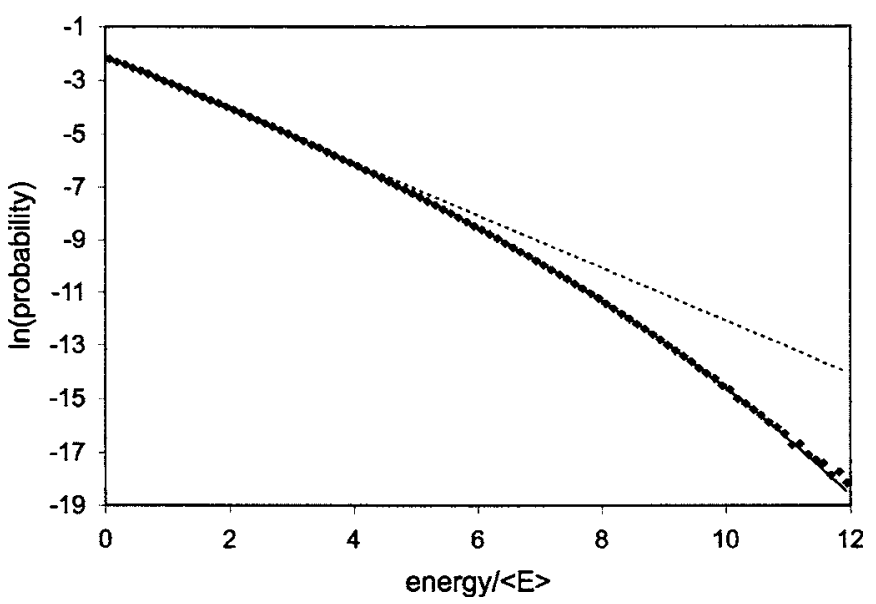

FIG. 1. The logarithm of the probability plotted vs one-particle kinetic energy divided by the one-particle average energy for a hard-sphere system of 20 particles in two dimensions with reflecting walls. The Boltzmann distribution is the dashed line. The NVE distribution of Eq. (9) is the solid line. The diamonds indicate simulation data of the time-weighted kinetic energy of particles in each of 96 intervals. In each case, the probability within the interval is integrated across the interval and plotted in the center of the interval. The correct distribution is considerably below the Boltzmann distribution at high energy.

$N V E$ energy distribution of Eq. (9) vanishes for $E_{1}>E$ and decays faster than the corresponding exponential as $E_{1}$ approaches $E$.

Related energy and velocity distributions have been described previously. Some have been limited to one dimension, for example, Olness and Hoover, ${ }^{21}$ Ackland, ${ }^{22}$ and Rouet et al. ${ }^{23}$ Nanbu et al. ${ }^{24}$ present simulation data for $N=2,3, \ldots, 10$ for three dimensions showing that Eq. (8) is correct. Others will be mentioned below.

Using Eq. (9), it is straightforward now to evaluate the average energy of particle 1 , as well as other energy moments, by converting the integral to an Euler integral of the first kind (beta function), ${ }^{25}$

$$
\begin{aligned}
\left\langle E_{1}^{k}\right\rangle_{N V E} & =\int_{0}^{E} P_{d}\left(N, V_{d}, E, E_{1}\right) E_{1}^{k} d E_{1} \\
& =\frac{\Gamma(d N / 2) \Gamma(d / 2+k)}{\Gamma(d / 2) \Gamma(d N / 2+k)} E^{k} \quad(\mathrm{hs}) .
\end{aligned}
$$

Equation (11) with $k=0$ shows that Eq. (9) is normalized. Using $k=1$ in Eq. (11) gives exact agreement with the equipartition theorem, $\left\langle E_{1}\right\rangle_{N V E}=E / N$; however, Eq. (11) with $k$ $\neq 0,1$ differs from the Boltzmann distribution, and the results of our simulations demonstrate the correctness of predicted moments.

Figure 1 plots the natural logarithm of the time-weighted single-particle kinetic energy probability in a simulation for the kinetic energy of any of $N=20$ identical particles with radius of 1 unit in a two-dimensional square box of edge length of 200 units with reflecting walls. This density corresponds to a low-density gas. The simulation included approximately $10^{9}$ collisions. We accumulated the timeweighted one-particle kinetic energy probability in 96 equalwidth intervals (bins) in the region of $0-12$ times the average energy. For two dimensions, the density of states is constant, 
so the Boltzmann distribution is exponential. The dashed line is the Boltzmann distribution integrated between the bin edges. The discrete points are connected to guide the eyes. The NVE energy distribution [Eq. (9)] was also integrated between the bin edges and the points connected with the solid line. The binned probabilities from the simulation are small diamonds. The difference between the $N V E$ distribution and the simulation data is distributed randomly within the expected statistical error (the standard deviation is less than twice the square root of the number of hits per bin). The statistical error increases to the right and is quite noticeable in the last few bins, which include less than 100 hits per bin. However, the number of hits in each of the first seven bins is over $10^{8}$, and these data are accurate to one part in $10^{4}$. This plot and the accumulated moments of this distribution as well as numerous other simulations confirm the accuracy of Eqs. (9) and (11).

\section{THE NVE VELOCITY DISTRIBUTION}

Probability distributions for velocity (speed) can be derived from those for momentum, Eq. (8), by substitution of $\boldsymbol{p}_{1}=m_{1} v_{1}$ and $d \boldsymbol{p}_{1}^{d}=2 \pi^{d / 2} p_{1}^{d-1} d p_{1} / \Gamma(d / 2)$ to obtain

$$
\begin{aligned}
P_{d}\left(N, V_{d}, E, v_{1}\right) d v_{1}= & \frac{2 \Gamma(d N / 2)}{\Gamma(d / 2) \Gamma(d(N-1) / 2)} \\
& \times\left(\frac{v_{1}}{v_{1 m}}\right)^{d}\left(1-\frac{v_{1}^{2}}{v_{1 m}^{2}}\right)^{d(N-1) / 2-1} \\
& \times \Theta\left(v_{1 m}^{2}-v_{1}^{2}\right) \frac{d v_{1}}{v_{1}} \quad(\mathrm{hs}),
\end{aligned}
$$

where $v_{1 m}=\left(2 E / m_{1}\right)^{1 / 2}$ is the maximum velocity possible for particle 1 (when all the other particles are motionless). Moments of the velocity distribution can also be evaluated by reducing the integral to an Euler integral of the first kind to obtain

$$
\begin{aligned}
\left\langle v_{1}^{k}\right\rangle_{N V E} & =\int_{0}^{v_{1 m}} P_{d}\left(N, V_{d}, E, v_{1}\right) v_{1}^{k} d v_{1} \\
& =\frac{\Gamma((d+k) / 2) \Gamma(d N / 2)}{\Gamma(d / 2) \Gamma((d N+k) / 2)} v_{1 m}^{k} \quad \text { (hs), }
\end{aligned}
$$

The $N V E$ average squared velocity, $\left\langle v_{1}^{2}\right\rangle_{N V E}$, from Eq. (13) gives the expected equipartition value, $2 E /\left(N m_{1}\right)$, but the $N V E$ average velocity, $\langle v\rangle_{N V E}$, is slightly higher than for an infinite system with a Maxwell-Boltzmann distribution of the same average energy,

$$
\begin{aligned}
\left\langle v_{1}\right\rangle_{N V E} & =\frac{\Gamma((d+1) / 2) \Gamma(d N / 2)}{\Gamma(d / 2) \Gamma((d N+1) / 2)}\left(\frac{2 E}{m_{1}}\right)^{1 / 2} \\
& =\left\langle v_{1}\right\rangle_{B}\left[1+\frac{1}{4 d N}+\frac{1}{32 d^{2} N^{2}}+O\left(N^{-3}\right)\right] \quad(\mathrm{hs}),
\end{aligned}
$$

$$
\left\langle v_{1}\right\rangle_{B}=\left(\frac{2 k_{B} T}{m_{1}}\right)^{1 / 2} \frac{\Gamma((d+1) / 2)}{\Gamma(d / 2)},
$$

and we have again defined the kinetic temperature using Eq. (7). The $N V E$ average velocity in Eq. (13) can differ from the Boltzmann value by a measurable amount for $N$ less than a few hundreds even though the rms velocity agrees exactly with the Boltzmann value. Moments of the momentum distribution can be obtained from Eq. (13) with suitable powers of $m_{1}$.

For later comparison, we calculate the average relative velocity between two independent particles in the $N V E$ ensemble using the two-particle velocity distribution. This is derived by integrating the phase space density over all of the coordinates and all of the momenta except for those of two particles, arbitrarily numbered particle 1 and particle 2 . The result is

$$
\begin{aligned}
P\left(N, V_{d}, E, \boldsymbol{p}_{1}, \boldsymbol{p}_{2}\right)= & \frac{\Gamma(d N / 2)}{\Gamma(d(N-2) / 2)} \\
& \times \frac{\left[1-\left[\left(\boldsymbol{p}_{1}^{2} / m_{1}+\boldsymbol{p}_{2}^{2} / m_{2}\right) / 2 E\right]\right]^{d(N-2) / 2-1}}{\left(m_{1} m_{2}\right)^{d / 2}(2 \pi E)^{d}} \\
& \times \Theta\left(E-\frac{\boldsymbol{p}_{1}^{2}}{2 m_{1}}-\frac{\boldsymbol{p}_{2}^{2}}{2 m_{2}}\right) \quad \text { (hs). }
\end{aligned}
$$

Using Eq. (16), we calculate moments of the average relative velocity of collisions between particle 1 and particle 2 by converting from momenta to velocities and to relative and center-of-mass coordinates. The result is

$$
\left\langle v_{\mathrm{rel}}^{k}\right\rangle_{N V E}=\frac{\Gamma((d+k) / 2) \Gamma(d N / 2)}{\Gamma(d / 2) \Gamma((d N+k) / 2)}\left(\frac{2 E}{\mu_{12}}\right)^{k / 2} \quad(\mathrm{hs}),
$$

where $\mu_{12}=m_{1} m_{2} /\left(m_{1}+m_{2}\right)$, the reduced mass of $m_{1}$ and $m_{2}$. Note that moments of the average relative velocity are obtained from moments of the average velocity [Eq. (13)] by simply replacing $m_{1}$ by $\mu_{12}$, the same as for the MaxwellBoltzmann distribution. This results in the following important ratio:

$$
\frac{\left\langle v_{\mathrm{rel}}^{k}\right\rangle_{N V E}}{\left\langle v_{1}^{k}\right\rangle_{N V E}}=\left(\frac{m_{1}+m_{2}}{m_{2}}\right)^{k / 2} \quad \text { (hs). }
$$

Even though Eq. (18) is the same as for the MaxwellBoltzmann distribution, this is an exact relationship for an arbitrary number of hard spheres in the $N V E$ ensemble using the exact $N V E$ hard-sphere velocity distribution. Our simulations confirm these analytic results.

Results in this section have exhibited distributions and moments of energy and speed that are different from those of the classical canonical ensemble. These can all be shown to limit to the canonical values as the system size increases. However, for finite systems, the introduction of momentum constraints, such as using periodic boundary conditions, alters these predictions in subtle and significant ways, even though they also limit to the same canonical results in the infinite system limit.

where 


\section{THE NVEPG ENSEMBLE}

To minimize wall effects, most molecular dynamics simulations are performed with periodic boundary conditions rather than reflecting walls. Even though $N, V_{d}$, and $E$ are still constant in these molecular dynamics implementations, additional conserved quantities alter the sampling of phase space from that of the microcanonical ensemble. In particular, in the absence of external fields, the total linear momentum and the (possibly uniformly moving) position of the center of mass are conserved by Newton's equations. Erpenbeck and Wood $^{5}$ named this the molecular dynamics ensemble even though the term does not seem have caught on. The constraints, $2 d$ in number, are

$$
P_{j}=\sum_{i=1}^{N} m_{i} v_{i j}(t) \quad \text { for } j=1,2, \ldots, d,
$$

for the $d$ components of the total momentum $\boldsymbol{P}$, where $v_{i j}(t)$ are the $j$ th velocity components of the $i$ th particle at time $t$, and the $d$ components of

$$
G_{j}=M_{\mathrm{tot}}^{-1}\left[P_{j} t-\sum_{i=1}^{N} m_{i}\left(r_{i j}(t)+\sigma_{i j}\right)\right] \text { for } j=1,2, \ldots, d,
$$

where $r_{i}(t)$ is the position of the $i$ th particle at time $t$, and $M_{\text {tot }}$ is the total mass of all $N$ particles. The position of the center of mass at time $t=0$ is $-\boldsymbol{G}$. The quantity $\boldsymbol{G}$ is defined by most authors without the overall division by $M_{\text {tot }}$ and, in that form, is the infinitesimal generator for Galilean frames of reference, ${ }^{26}$ however, we include the divisor $M_{\text {tot }}$ in Eq. (20) to define the center-of-mass position itself. The $d$-dimensional vector $\boldsymbol{\sigma}_{i}$ is a repeating-cell lattice vector defining the cell to which the $i$ th particle has moved in time $t$. If this vector is not included, $\boldsymbol{G}$ would be discontinuous whenever a particle moved across a cell boundary. ${ }^{5}$

These constraints change the phase space density, the integrand of Eq. (2), from $h^{-d N} \delta(E-\boldsymbol{H})$ to

$$
\begin{aligned}
& h^{-d(N-1)} \delta(E-\boldsymbol{H}) \delta\left(\boldsymbol{P}-\sum_{i=1}^{N} \boldsymbol{p}_{i}\right) \\
& \quad \times \delta\left(\boldsymbol{G}-M_{\mathrm{tot}}^{-1}\left[\boldsymbol{P} t-\sum_{\sigma} \sum_{i=1}^{N} m_{i} \boldsymbol{r}_{i}(t)\right]\right),
\end{aligned}
$$

where the vector argument for $\delta(x)$ implies a product of $d \delta$ functions of the components. The NVEPG ensemble density of states is thus given by

$$
\begin{aligned}
G_{d}\left(N, V_{d}, E, \boldsymbol{P}, \boldsymbol{G}\right)= & h^{-d(N-1)} \int \delta(E-\boldsymbol{H}) \delta\left(\boldsymbol{P}-\sum_{i=1}^{N} \boldsymbol{p}_{i}\right) \\
& \times \delta\left(\boldsymbol{G}-M_{\mathrm{tot}}^{-1}\left[\boldsymbol{P} t-\sum_{\sigma} \sum_{i=1}^{N} m_{i} \boldsymbol{r}_{i}(t)\right]\right) \\
& \times d q^{d N} d p^{d N} .
\end{aligned}
$$

Historically, the preceding equations were first developed using the conservation of $\boldsymbol{P},{ }^{5,9,12,16,27,28}$ and the conservation of $\boldsymbol{G}$ was added later when its importance was realized. ${ }^{11,26,29}$
Equation (22) can be evaluated using the Laplace transform form of $\delta(E-\boldsymbol{H})$ and the Fourier transform form of the momentum $\delta$-function constraints. ${ }^{13,17}$ The analysis is simpler in Jacobi coordinates. ${ }^{26,29}$ Çagin and Ray ${ }^{9}$ and Ray and Zhang ${ }^{26}$ demonstrated how to incorporate the effect of the center-ofmass position constraint by showing that it eliminates one factor of $V_{d}$ representing the integration over the center-ofmass coordinates. If we again assume impenetrable bodies, then using methods similar to those of Çagin and Ray, ${ }^{9}$ Román et al. ${ }^{19,27}$ and Lado, ${ }^{16}$ we obtain

$$
\begin{aligned}
G_{d}\left(N, V_{d}, E, \boldsymbol{P}, \boldsymbol{G}\right)= & \frac{(2 \pi)^{d(N-1) / 2}\left(Z\left(N, V_{d}\right) / V_{d}\right)}{h^{d(N-1)} \Gamma(d(N-1) / 2) M_{\mathrm{tot}}^{d / 2}}\left(\prod_{i=1}^{N} m_{i}^{d / 2}\right) \\
& \times\left(E-\frac{\boldsymbol{P}^{2}}{2 M_{\mathrm{tot}}}\right)^{d(N-1) / 2-1} \\
& \times \Theta\left(E-\frac{\boldsymbol{P}^{2}}{2 M_{\mathrm{tot}}}\right) \quad(\mathrm{hs}) .
\end{aligned}
$$

These expressions are applicable if $N \geqslant 3$. Using Eq. (23) with Eq. (3) in analogy with Eqs. (4) and (5) and $S_{\text {NVEPG }}$ $=k_{B} \ln \Omega_{d}$, we obtain the kinetic definition of temperature in the NVEPG ensemble, ${ }^{9,19,26}$

$$
\langle K\rangle_{N V E P G}=\frac{d}{2}(N-1) k_{B} T+\frac{\boldsymbol{P}^{2}}{2 M_{\mathrm{tot}}},
$$

where the total momentum $\boldsymbol{P}$ is usually set to be zero. For hard spheres, Eq. (24) is the total energy $E$ in analogy with Eqs. (6) and (7). In the case of $\boldsymbol{P}=0, N$ particles in the NVEPG ensemble with periodic boundary conditions will have a higher temperature than $N$ particles with the same initial conditions with reflecting boundary conditions ( $N V E$ ensemble). This is because the molecular dynamics simulation with periodic boundary conditions has $d$ fewer degrees of freedom among which to distribute its energy. As shown below, the reverse side of this fact is that individual particle energies are limited to a maximum energy that is lower than the total system energy. It is important to note that Eq. (24) is an exact definition of $T$ for $N=3,4, \ldots$, not just in the thermodynamic limit.

Integrating the phase space density, Eq. (21), over all the coordinates and all the momenta except those of particle 1, using methods similar to Lado ${ }^{16}$ and Ray and Zhang, ${ }^{26}$ we obtain the one-particle momentum distribution,

$$
\begin{aligned}
P_{d}\left(N, V_{d}, E, \boldsymbol{P}, \boldsymbol{G}, \boldsymbol{p}_{1}\right) d \boldsymbol{p}_{1}^{d}= & \frac{\Gamma(d(N-1) / 2)}{\Gamma(d(N-2) / 2)\left(2 \pi \mu_{1} E_{\text {c.m.m. }}\right)^{d / 2}} \\
& \times\left(1-\frac{\boldsymbol{p}_{1 \text { c.m. }}^{2}}{2 \mu_{1} E_{\text {c.m. }}}\right)^{[d(N-2) / 2]-1} \\
& \times \Theta\left(E_{\text {c.m. }}-\frac{\boldsymbol{p}_{1 \text { c.m. }}^{2}}{2 \mu_{1}}\right) d \boldsymbol{p}_{1}^{d} \quad(\mathrm{hs}),
\end{aligned}
$$

where 


$$
\boldsymbol{p}_{1 \mathrm{c} . \mathrm{m} .}=\boldsymbol{p}_{1}-\boldsymbol{P} \frac{m_{1}}{M_{\mathrm{tot}}}, \quad E_{\mathrm{c} . \mathrm{m} .}=E-\frac{\boldsymbol{P}^{2}}{2 M_{\mathrm{tot}}},
$$

and

$$
\mu_{1}=\frac{m_{1}\left(M_{\mathrm{tot}}-m_{1}\right)}{M_{\mathrm{tot}}}
$$

In this set of equations, $p_{1 \text { c.m. }}$ is the first particle's momentum shifted by its weighted share of the center-of-mass momentum, i.e., $\boldsymbol{p}_{1}$ in the center-of-mass frame, $E_{\text {c.m. }}$ is the total energy in the center-of-mass frame, and $\mu_{1}$ is the reduced mass of particle 1 and the rest of the system. The appearance of $\mu_{1}$ in Eq. (25) means that the maximum kinetic energy of particle 1 in the center-of-mass frame is $E_{m 1}=E\left(M_{\text {tot }}\right.$ $\left.-m_{1}\right) / M_{\text {tot }}$ rather than the total energy, as it is in the $N V E$ ensemble. The maximum energy occurs when particle 1 is moving in one direction with maximum energy and the other $N-1$ particles are moving in the opposite direction with the minimum energy needed to offset the momentum of particle 1. Note that Eq. (25) is the same as Eq. (8) with $p_{1}$ replaced by $p_{1 \text { c.m. }}, E$ replaced by $E_{\text {c.m. }}$, and $m_{1}$ replaced by $\mu_{1}$. Lado ${ }^{16}$ derived Eq. (25) for the case of identical particles. Rouet et $a l^{23}$ compared velocity distributions for both reflecting and periodic boundaries for $N=3-5$ from one-dimensional simulations. Román et al. ${ }^{19}$ displayed momentum distributions from two-dimensional simulations for $N=3,4,6$, and 10 and compared them with the theoretical distributions [Eq. (25)]. They also displayed the effect of a nonzero value of $\boldsymbol{P}$. Reference 19 also devotes extensive effort to expressing the momentum distribution in the original reference frame. The formulas simplify considerably in the center-of-mass frame as we have displayed them. The difference between Eq. (25) above and Eq. (69) of Ref. 19, which is an intermediate step in arriving at the form of Eq. (25), deserves comment. First, our derivation is more general in allowing unequal masses. Second, we have completed the square in $p_{1 \text { c.m. }}$ so $p_{1}$ appears only once inside the large parentheses. This operation allows easy identification of the useful quantities in the expression. We learned the utility of this step from Lado's derivation. ${ }^{16}$

Converting from a momentum distribution to an energy distribution as before, we obtain the one-particle energy distribution in the NVEPG ensemble,

$$
\begin{aligned}
P_{d}\left(N, V_{d}, E, \boldsymbol{P}, \boldsymbol{G}, E_{1}\right) d E_{1}= & \frac{\Gamma(d(N-1) / 2)}{\Gamma(d / 2) \Gamma(d(N-2) / 2)}\left(\frac{E_{1}}{E_{m 1}}\right)^{d / 2} \\
& \times\left(1-\frac{E_{1}}{E_{m 1}}\right)^{[d(N-2) / 2]-1} \\
& \times \Theta\left(E_{m 1}-E_{1}\right) \frac{d E_{1}}{E_{1}} \quad(\mathrm{hs}),
\end{aligned}
$$

where

$$
E_{1}=\frac{\left(\boldsymbol{p}_{1}-\boldsymbol{P}\left(m_{1} / M_{\mathrm{tot}}\right)\right)^{2}}{2 m_{1}}
$$

and

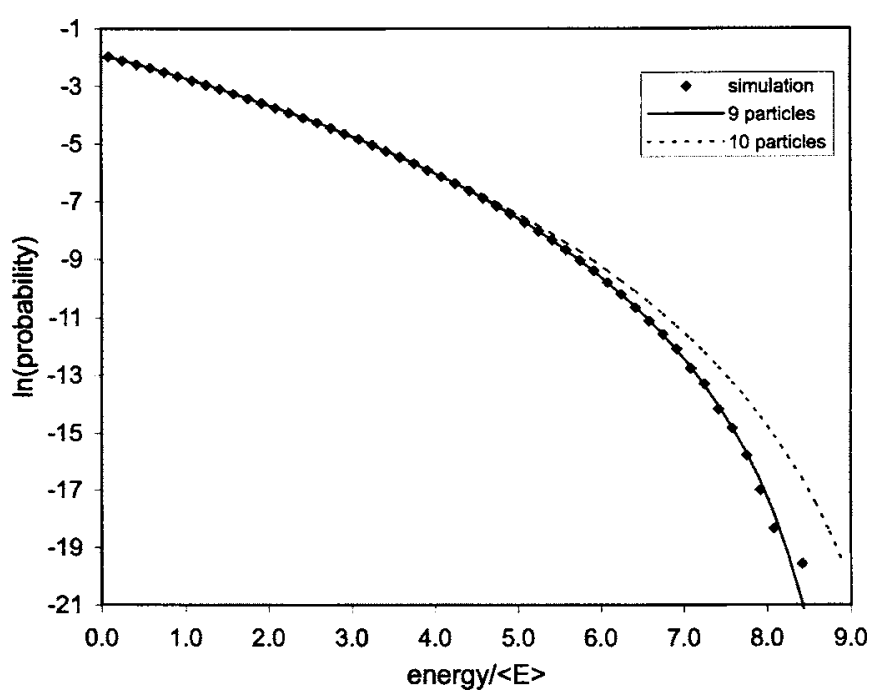

FIG. 2. The effect of periodic boundary conditions on the hard-sphere oneparticle energy distribution is demonstrated. The diamonds show simulation data for two dimensions and $N=10$. The distribution for ten particles using Eq. (9) extrapolates to a zero at ten times the average energy. The actual data are fitted to within statistical error by Eq. (9) with $N$ replaced by $N-1$ and $E$ replaced by $9 E / 10$ as required by the center-of-mass constraint [Eq. (27)].

$$
E_{m 1}=\left(E-\frac{\boldsymbol{P}^{2}}{2 M_{\mathrm{tot}}}\right) \frac{\left(M_{\mathrm{tot}}-m_{1}\right)}{M_{\mathrm{tot}}} \quad(\mathrm{hs}) .
$$

Comparing Eq. (27) with Eq. (9) and $N V E$ ensemble results of Secs. II and III for the energy moments, momentum, or velocity distributions reveals two changes summarized by the following substitutions: $N \rightarrow N-1$ and $E \rightarrow E_{m 1}$. Thus, $N V E$ formulas exhibited earlier can be used in the NVEPG ensemble by making these substitutions. In particular, the energy moments are given by

$$
\begin{aligned}
\left\langle E_{1}^{k}\right\rangle_{N V E \boldsymbol{P G}} & =\int_{0}^{E_{m 1}} P_{d}\left(N, V_{d}, E, \boldsymbol{P}, \boldsymbol{G}, E_{1}\right) E_{1}^{k} d E_{1} \\
& =\frac{\Gamma(d / 2+k) \Gamma(d(N-1) / 2)}{\Gamma(d / 2) \Gamma(d(N-1) / 2+k)} E_{m 1}^{k} \quad \text { (hs). }
\end{aligned}
$$

If $k=1$, the average energy in the center-of-mass frame, $E_{m 1} /(N-1)$, is mass dependent [see Eq. (28)]. This represents a breakdown of the equipartition theorem for particles of different masses. Heavy particles have a lower average kinetic energy than light particles. This arises because the center-of-mass constraint (a momentum constraint) affects the kinetic energy of heavy and light particles differently. This effect was noted by Ackland ${ }^{22}$ for one-dimensional systems, but this analysis establishes the principle for arbitrary dimension. Again, breakdown of the equipartition theorem is inversely proportional to the size of the system,

$$
\frac{\langle E\rangle_{N V E \boldsymbol{P G}, \text { heavy }}}{\langle E\rangle_{N V E \boldsymbol{P G}, \text { light }}}=1-\frac{\left(m_{\text {heavy }}-m_{\text {light }}\right)}{M_{\text {tot }}}+O\left(M_{\text {tot }}^{-2}\right) \quad \text { (hs) } \text {. }
$$

We emphasize, however, that this breakdown of the equipartition principle is not only seen in the average energy, but in the entire one-particle energy distribution [Eq. (28)] as discussed below regarding Fig. 3. 


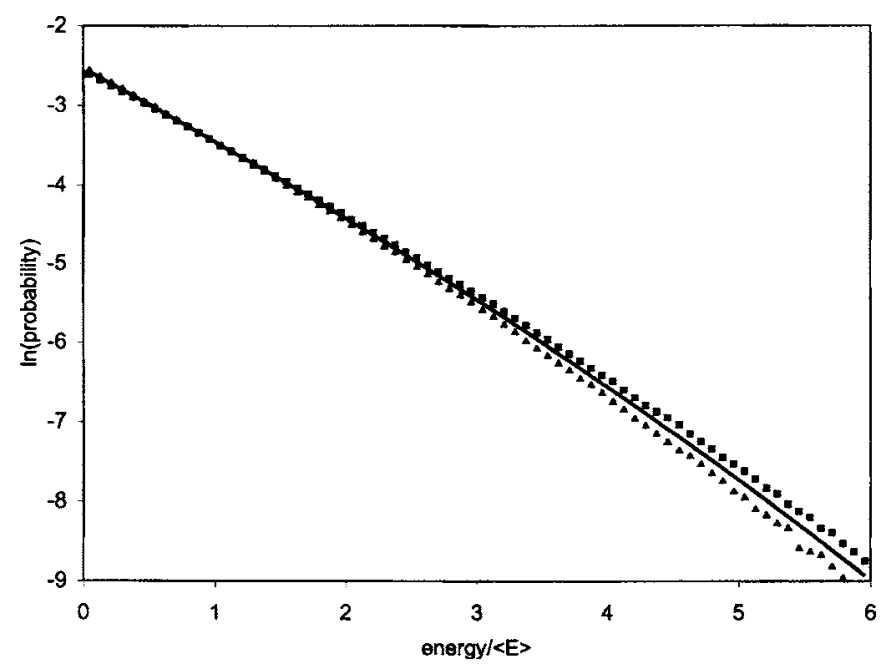

FIG. 3. Shows the breakdown of the equipartition theorem for periodic boundary conditions and unequal masses. The data show one-particle kinetic energy probabilities as a function of energy for ten particles of mass of 1 (squares) and ten particles of mass of 4 (triangles). The solid line is the energy distribution if all particles had the same mass. The squares are fitted within statistical error by a curve with average energy of $98\langle E\rangle / 95$ and the triangles by a curve with average energy $92\langle E\rangle / 95$ as predicted by Eqs. (27) and (28).

The replacement of $N$ by $N-1$ in the NVEPG ensemble can be viewed as due to a change in the definition of temperature [Eq. (24)], whereas the replacement of $E$ by $E_{m 1}$ is due to the momentum constraint limiting the maximum velocity. For identical particles, these two factors identically offset each other. The first factor raises the average energy by $N /(N-1)$ because of the new definition of temperature, and the second reduces the maximum energy by $(N-1) / N$.

Figure 2 shows the binned one-particle energy distribution from a two-dimensional simulation with $N=10, \boldsymbol{P}=0$, and $10^{8}$ collisions. The average energy of each particle is one-tenth of the total energy. The dotted line is the distribution expected for ten particles (NVE ensemble) [Eq. (9)]. The solid line is the $N V E$ distribution for nine particles and a total energy given by $9 E / 10$, equivalent to Eq. (27). At high energy, it is clear that the correct distribution is the solid line, which goes to zero (logarithm goes to $-\infty$ ) at nine times the average energy. In contrast, the distribution given by the dotted line goes to zero at ten times the average energy, a one-particle energy forbidden by the center-of-mass constraint.

Figure 3 illustrates the breakdown of the equipartition theorem for particles with unequal mass. These data are taken from a simulation with ten particles of mass of 1 (arbitrary units) and ten particles of mass of 4 in two dimensions. The squares are the binned probabilities for the light particles, and the triangles are the binned probabilities of the heavy particles. The solid line is the distribution of kinetic energy predicted from the average energy of the system (taken to be unity here). It is easy to see that heavy and light particles have different distributions. Equation (29) predicts that the light particles should have an average energy of $98 / 95 \approx 1.03158$, and the heavy particles should have an average energy of $92 / 95 \approx 0.96842$. The simulation results are 1.03124 and 0.96872 , respectively, within the expected statistical error. This complication has probably not been previously detected because analytic work has focused on identical particles.

The $k$ th velocity moment has the following value in the NVEPG ensemble:

$$
\begin{aligned}
\left\langle v_{1}^{k}\right\rangle_{N V E \boldsymbol{P G}} & =\int_{0}^{v_{1 m M D}} P_{d}\left(N, V_{d}, E, \boldsymbol{P}, \boldsymbol{G}, v_{1}\right) v_{1}^{k} d v_{1} \\
& =\frac{\Gamma((d+k) / 2) \Gamma(d(N-1) / 2)}{\Gamma(d / 2) \Gamma([d(N-1)+k] / 2)} v_{1 m M D}^{k} \quad(\mathrm{hs})
\end{aligned}
$$

where $\nu_{1 m M D}=\left[2 E_{\mathrm{c} . \mathrm{m} .}\left(M_{\mathrm{tot}}-m_{1}\right) / m_{1} M_{\mathrm{tot}}\right]^{1 / 2}$ is the maximum velocity allowed by the center-of-mass constraint for a particle of mass $m_{1}$ in the center-of-mass frame. Compared to Eq. (13), the same two factors tend to offset each other here, too: the replacement of $N$ by $N-1$ in the gamma function factor representing the change in temperature definition and the replacement of $\nu_{1 m}$ by $\nu_{1 m M D}$ representing the change in maximum allowed velocity.

The average relative velocity between two colliding particles in the NVEPG ensemble requires additional attention. This quantity is required because the collision rate is a product of three factors: density of collision partners, cross section for collision, and average relative velocity. When particle 1 is moving in a given direction, on the average, the other particles are moving in the opposite direction. Thus, the center-of-mass constraint increases the relative velocity of collisions above what it would be based on the average velocity. ${ }^{30}$ To calculate this, we evaluate the two-particle momentum distribution by integrating the phase density [Eq. (21)] over all the coordinates and over all the momenta except those of particle 1 and particle 2 . The result is

$$
\begin{aligned}
P_{d M D}\left(N, V_{d}, E, \boldsymbol{P}, \boldsymbol{G}, \boldsymbol{p}_{1}, \boldsymbol{p}_{2}\right)= & \frac{\Gamma(d(N-1) / 2)}{\Gamma(d(N-3) / 2)\left(2 \pi m_{\mathrm{eff}} E_{\mathrm{c} . \mathrm{m} .)^{d}} \Theta\left(E_{\mathrm{c} . \mathrm{m} .}-E_{12}\right)\right.} \\
& \times\left\{\begin{array}{l}
\left.\frac{\left(m_{2} \boldsymbol{p}_{1}-m_{1} \boldsymbol{p}_{2}\right)^{2}}{2 m_{1} m_{2}\left(m_{1}+m_{2}\right)}+\frac{M_{\mathrm{tot}}\left[\boldsymbol{p}_{1}+\boldsymbol{p}_{2}-\frac{\left(m_{1}+m_{2}\right) \boldsymbol{P}}{2\left(m_{1}+m_{2}\right)\left(M_{\mathrm{tot}}-m_{1}+m_{2}\right)}\right.}{E_{\mathrm{c} . \mathrm{m} .}}\right\} \\
\text { (hs }),
\end{array}\right.
\end{aligned}
$$


where $E_{12}$ is the expression above $E_{\mathrm{c} . \mathrm{m} .}$ in the last line of Eq. (32) and where

$$
m_{\mathrm{eff}}=\left(\frac{m_{1} m_{2}\left(M_{\mathrm{tot}}-m_{1}-m_{2}\right)}{M_{\mathrm{tot}}}\right)^{1 / 2} .
$$

We first converted this distribution to a velocity distribution and then to center-of-mass and relative velocities for particles 1 and 2. The resulting distribution was multiplied by $\nu_{\text {rel }}^{k}$ and the result integrated over the center-of-mass and relative coordinates. The resulting average relative velocity moments are

$\left\langle v_{\mathrm{rel}}^{k}\right\rangle_{N V E \boldsymbol{P G}}=\frac{\Gamma((d+k) / 2) \Gamma(d(N-1) / 2)}{\Gamma(d / 2) \Gamma((d(N-1)+k) / 2)}\left(\frac{2 E_{\mathrm{c} . \mathrm{m} .}}{\mu_{12}}\right)^{k / 2}$

The important fact quantified by Eq. (34) is that even though the average velocity is reduced by the appearance of the factor of $\left[\left(M_{\mathrm{tot}}-m_{1}\right) / M_{\mathrm{tot}}\right]^{1 / 2}$ in $\nu_{1 m M D}$ in Eq. (31), the average relative velocity is not. It is only reduced by the replacement of $N$ by $N-1$ in the gamma function prefactor. The NVEPG ensemble ratio comparable to Eq. (18) is thus given by

$$
\frac{\left\langle v_{\mathrm{rel}}^{k}\right\rangle_{N V E \boldsymbol{P G}}}{\left\langle v_{1}^{k}\right\rangle_{N V E \boldsymbol{P G}}}=\left(\frac{M_{\mathrm{tot}}\left(m_{1}+m_{2}\right)}{m_{2}\left(M_{\mathrm{tot}}-m_{1}\right)}\right)^{k / 2} \quad \text { (hs). }
$$

Comparing Eq. (35) to Eq. (18), the ratio of the average relative velocity to the average velocity for identical particles with periodic boundary conditions is increased by a factor of $[N /(N-1)]^{1 / 2}$ over a system with reflecting walls. This change for periodic boundary conditions is apparent in our hard-sphere simulations in the computed collision rate, the mean free path, and the equation of state. Predicted values of Equation (34) are quantitatively reproduced in our simulations for $d=2$, and 3 with equal masses and for $d=1,2$, and 3 for unequal masses ( $d=1$ with equal masses is not ergodic). Erpenbeck and Wood ${ }^{5}$ previously established Eq. (34) for $k$ $=1$ [their Eq. (47)] for the $N V E \boldsymbol{P}(\boldsymbol{G})$ ensemble for identical particles by a different method, but they did not comment on the relationship of the average relative velocity to the average velocity nor point out its significance for unequal masses.

Note also that the average relative collision energy, $\mu_{12} \nu_{\mathrm{rel}}^{2} / 2$, using $k=2$ in Eq. (35), is raised by a factor of $N /(N-1)$ for identical particles. This change is important in studying reacting systems. However, the distribution of relative collision energies falls to zero faster in a finite system than the Boltzmann distribution, so even if the average relative collision energy is slightly higher, the probability of having enough relative kinetic energy to surmount a high barrier would be smaller than for an infinite system (see Figs. 1-3). All these corrections should be understood when extrapolating finite system simulation results to predict the properties of macroscopic systems.

\section{DISCUSSION AND CONCLUSION}

All the results derived here have been verified to be correct by numerous hard-sphere simulations. Relative devia- tions from the expressions were always within the statistical uncertainty, which was taken to be one or two times the reciprocal of the square root of the number of collisions.

We chose to display results from two-dimensional simulations because the density of states is constant, so the Boltzmann distribution is a straight line in a logarithmic plot. For one-dimensional hard-sphere simulations, the probability distribution for energy has a square root singularity at low energy. In three dimensions, the probability distribution for energy rises as $E_{1}^{1 / 2}$, reaches a maximum, and then falls. These characteristics complicate the presentation of data, and so simulation data have not been included in this paper for $d=1$ or $d=3$. However, numerous additional examples are displayed online. ${ }^{7}$

At first, we thought that the kinetic energy distribution given by Eq. (9) would be correct for all microcanonical systems (and with suitable substitution, for molecular dynamics systems), independent of the interparticle potential. However, the microcanonical momentum integral and coordinate integral of Eq. (4) do not factor into separate factors like they do in the canonical ensemble except for nonpenetrating particles (of which hard spheres is one example), thus allowing the simplification to Eq. (5). Because of this nonseparability, the microcanonical kinetic energy distribution for attractive particles is more complicated than Eq. (9). Consider, for example, a small number of particles interacting through a Lennard-Jones or other similar two-body potential. When the particles are well separated, the total kinetic energy and total energy would be approximately equal like in the hard-sphere system. When two particles become bound together through a collision, their potential energy becomes negative. The corresponding excess kinetic energy is carried off by a third particle involved in the collision. This increases the available kinetic energy, allowing the maximum kinetic energy of the remaining particles to increase to a value above the total system energy. The kinetic energy distribution, and consequently the velocity distribution, would be a weighted average of functions like Eq. (9) with different total kinetic energies. The weighting of total kinetic energy values will depend on the total energy. It is not clear how the resulting distribution will differ from a Boltzmann distribution. We have not yet done this experiment, but we expect some carryover of the effects seen in hard-sphere simulations into more realistic molecular dynamics.

Molecular dynamics simulation is usually performed to model behavior occurring in bulk material. However, the extrapolation of simulation results to bulk matter must be done carefully. The finite size effects presented here should be considered and appropriate corrections applied. Temperature, average velocity, average relative velocity, average collision energy, and the probability of surmounting a barrier are all affected by the size of the system simulated. One can try to avoid these difficulties by increasing the size of the system. However, finite-system corrections for a larger system, albeit smaller, would still be needed. Furthermore, since simulation time and required resources often increase dramatically as the system size increases, it may be more effective to apply appropriate corrections to a smaller system.

Modern molecular dynamics simulations are often done 
now using thermostats for constant temperature and/or barostats for constant pressure. Using these techniques might reduce the effects described here. However, since these effects follow from equations of motion and quantities conserved by the equations of motion, they will appear to some degree in all molecular dynamics simulations. For example, it should not be surprising to find that the velocity distribution in a simulation is non-Boltzmann. ${ }^{17}$ Deviations from a Boltzmann distribution may be partially due to the size of the system. Understanding the effects of system size should help interpret any non-Boltzmann distributions and to extrapolate results to correct bulk values.

Monte Carlo techniques are complementary, and sometimes faster, for obtaining equilibrium properties of systems. There are now well-developed methods ${ }^{28,31,32}$ for sampling phase space with the proper statistical weights to produce results for direct comparison with molecular dynamics simulations. On the other hand, if standard canonical ensemble Monte Carlo methods are used, corrections of the order of $N^{-1}$ are again needed to make relevant comparisons.

For experimental phenomena in isolated, finite systems such as cluster dynamics, unimolecular reactions, or nuclear decay, finite system size corrections are required for accurate theory. For this reason, microcanonical theory is required to accurately describe unimolecular reaction rates. ${ }^{33}$ The probability of surmounting a potential energy barrier in a unimolecular reaction is smaller than that of a system assumed to be described by a Boltzmann distribution. Continued developments in the statistical mechanics of finite systems will be required to accurately describe the behavior of finite systems as scientists continue to do experiments on systems of smaller and smaller size.

The notable results of this paper are threefold. First, we highlighted the differences between the one-particle energy, momentum, and velocity distributions in the $N V E$ ensemble compared to the NVEPG ensemble and compared to the canonical ensemble (Boltzmann distribution). Some of these have been described in previous work, but our derivation for particles of nonidentical masses and arbitrary dimension is new and allows the further conclusions. As part of this comparison, we exhibited formulas for arbitrary moments of energy, momentum, velocity, and relative velocity, commenting on how they differ from the Boltzmann distribution and approach it as the number of particles increases. Second, we identified, quantified, and explained the breakdown of the equipartition theorem in the NVEPG ensemble for particles of unequal mass. This is caused by the center-of-mass constraint inherent in periodic boundary conditions for a finite system. This understanding is only made possible by the detailed comparison of the corresponding distributions and their moments for the two ensembles. It is perhaps ironic that using periodic boundary conditions to make the system simpler and the apparent spatial extent larger actually makes the system behave like a kinematically smaller system and creates complexities in interpreting the results. Third, from the calculated moments, we showed how the ratio of the average relative velocity to the average velocity and the ratio of relative kinetic energy to the average kinetic energy in the
NVEPG ensemble are also changed from values in the NVE ensemble, again due to the center-of-mass constraint. These quantities are important in studying collision kinetics and reaction rates in finite systems.

\section{ACKNOWLEDGMENTS}

Computations for this work were performed, in part, using resources of the Ira and Mary Lou Fulton Supercomputer Center at Brigham Young University. Two of the authors (S.R.B. and A.M.J.) thank Brigham Young University for Undergraduate Research Awards. The authors thank Douglas J. Henderson and Michael R. Shirts for useful comments and discussion.

${ }^{1}$ R. B. Shirts, BOLTZMANN, a kinetic molecular theory demonstrator, Trinity Software, Campton, NH, 1995.

${ }^{2}$ R. B. Shirts, S. R. Burt, and B. Lemmon, BoltzmanN 3D, available at http://people.chem.byu.edu/rbshirts/research/boltzmann_3d/

${ }^{3}$ R. B. Shirts and M. R. Shirts, J. Chem. Phys. 117, 5564 (2002).

${ }^{4}$ J. L. Lebowitz and J. K. Percus, Phys. Rev. 124, 1673 (1961); J. L. Lebowitz, J. K. Percus, and L. Verlet, ibid. 153, 250 (1967).

${ }^{5}$ J. J. Erpenbeck and W. W. Wood, in Statistical Mechanics, Part B: Timedependent Processes, Modern Theoretical Chemistry Vol. 6, edited by B. J. Berne (Plenum, New York, 1977), pp. 1-40.

${ }^{6}$ M. P. Allen and D. J. Tildesley, Computer Simulation of Liquids (Clarendon, Oxford, 1987), pp. 101-105.

${ }^{7} \mathrm{http} / / /$ people.chem.byu.edu/rbshirts/research/distributionexamples.pdf

${ }^{8}$ A. Schlüter, Z. Naturforsch. A 3, 350 (1948); an english translation of this article is found at http://people.chem.byu.edu/rbshirts/research/ schluter1948translation.doc

${ }^{9}$ T. Çagin and J. R. Ray, Phys. Rev. A 37, 247 (1988).

${ }^{10}$ K. Huang, Statistical Mechanics, 2nd ed. (Wiley, New York, 1987), p. 134.

${ }^{11}$ W. W. Wood, J. J. Erpenbeck, G. A. Baker, Jr., and J. D. Johnson, Phys. Rev. E 63, 011106 (2000).

${ }^{12}$ R. Lustig, J. Chem. Phys. 100, 3048 (1994).

${ }^{13}$ E. M. Pearson, T. Halicioglu, and W. A. Tiller, Phys. Rev. A 32, 3030 (1985).

${ }^{14}$ For example, D. A. McQuarrie, Statistical Mechanics (Harper \& Row, New York, 1976), p. 58.

${ }^{15}$ A. I. Khinchin, Mathematical Foundations of Statistical Mechanics (Dover, New York, 1949), p. 102; the first derivation of the volume and surface area of a many dimensional sphere known to us dates from 1902 [J. W. Gibbs, Elementary Principles of Statistical Mechanics (Dover, New York, 1960), p. 93].

${ }^{16}$ F. Lado, J. Chem. Phys. 75, 5461 (1981).

${ }^{17}$ J. R. Ray and H. W. Graben, Phys. Rev. A 44, 6905 (1991).

${ }^{18}$ When $d N$ is odd, the contour cannot be closed because of the branch cut along the negative real axis, but the contour can be distorted to a path wrapping around the branch cut, obtaining the same result.

${ }^{19}$ F. L. Román, A. Gonzalez, J. A. White, and S. Velasco, Physica A 234, 53 (1996).

${ }^{20} \mathrm{~S}$. Velasco, J. A. White, and J. Güémez, Eur. J. Phys. 14, 166 (1993).

${ }^{21}$ R. J. Olness and W. J. Hoover, Am. J. Phys. 42, 584 (1974).

${ }^{22}$ F. J. Ackland, Phys. Rev. E 47, 3268 (1993).

${ }^{23}$ J. L. Rouet, F. Blasco, and M. R. Feix, J. Stat. Phys. 71, 29 (1993).

${ }^{24}$ K. Nanbu, K. Iwata, and M. Hasegawa, Transp. Theory Stat. Phys. 23, 1071 (1994).

${ }^{25}$ I. S. Gradshteyn and I. M. Rhzhik, Table of Integrals, Series and Products (Academic, New York, 1965), formulas 8.380.1 and 8.384.1.

${ }^{26}$ J. R. Ray and Z. H. Zhang, Phys. Rev. E 59, 4781 (1999).

${ }^{27}$ F. L. Román, J. A. White, and S. Velasco, Phys. Rev. E 51, 6271 (1995).

${ }^{28}$ D. C. Wallace and G. K. Straub, Phys. Rev. A 27, 2201 (1983).

${ }^{29}$ K. Maier and S. Kabelac, J. Chem. Phys. 124, 064104 (2006).

${ }^{30}$ F. O. Raineri and H. L. Friedman, J. Chem. Phys. 91, 5642 (1989).

${ }^{31}$ R. Lustig, J. Chem. Phys. 109, 8816 (1998).

${ }^{32}$ M. A. Carignano, Commun. Pure Appl. Math. 361, 291 (2002).

${ }^{33}$ T. Baer and W. L. Hase, Unimolecular Reaction Dynamics (Oxford University Press, New York, 1996). 